\title{
A REDUCTION FORMULA
}

\author{
by G. N. WATSON \\ (Received 11th November, 1953)
}

1. In this paper I construct a reduction formula for the integral

$$
I_{n} \equiv \int_{0}^{1} x^{\alpha}(1-x)^{\beta} \frac{d^{n}\left\{x^{\gamma}(1-x)^{\delta}\right\}}{d x^{n}} d x
$$

the formula connects any three consecutive members of a set $I_{0}, I_{1}, I_{2}, \ldots, I_{m}$. We regard $m$ as given, and, in order to avoid wasting time over trivialities, we postulate that the constants $\alpha, \beta, \gamma, \delta$ (which are not restricted to be real) have real parts large enough to ensure (i) that the integrals $I_{n}$ under consideration and the integrals related to them which will be introduced subsequently are all absolutely convergent, and (ii) that, in all the partial integrations which will be effected, the integrated parts vanish at both limits.

The limitations on the constants can be avoided by using double circuit integrals of Pochhammer's type instead of definite integrals, but it seems desirable to avoid the com. plexities thereby introduced.

The integrals $I_{n}$ seem to be of some intrinsic importance; they are readily expressible in terms of the general terminating hypergeometric series of the type ${ }_{3} F_{2}$ with last element unity, so that the reduction formula may be regarded as a relation connecting three contiguous functions of this type ; and the reduction formula may be regarded as a soluble linear difference equation of the second order with a non-trivial solution, so that it augments the scanty number of such equations hitherto known.

We observe that

and also that

$$
I_{0}=\frac{\Gamma(\alpha+\gamma+1) \Gamma(\beta+\delta+1)}{\Gamma(\alpha+\beta+\gamma+\delta+2)}
$$

$$
I_{1}=\frac{\Gamma(\alpha+\gamma) \Gamma(\beta+\delta)}{\Gamma(\alpha+\beta+\gamma+\delta+1)}(\beta \gamma-\alpha \delta) ;
$$

it is also to be remarked that $I_{n}$ vanishes for odd values of $n$ in two special cases, namely (i) $\alpha=\gamma$ and $\beta=\delta$, and (ii) $\alpha=\beta$ and $\gamma=\delta$; (i) can be proved by effecting $n$ partial integrations while (ii) can be proved by writing $1-x$ in place of the dummy variable $x$. Strangely enough, both of these special cases occurred in a problem about Legendre polynomials which I have recently been studying, and, by keeping them in mind, I have been enabled to simplify the presentation of parts of the following work. In these special cases, the reduction formula yields simple expressions for $I_{n}$ (with $n$ even) in terms of Gamma functions.

2. The first stage in the work consists in constructing a linear differential equation of the second order satisfied by the integrand in $I_{n}$. We write

$$
x^{\alpha}(1-x)^{\beta} \frac{d^{n}\left\{x^{\gamma}(1-x)^{\delta}\right\}}{d x^{n}} \equiv y_{n}
$$

and, in order to construct the equation with a minimum of calculation, we observe that, if $x^{\gamma}(1-x)^{\delta} \equiv z$, then

$$
x(1-x) \frac{d z}{d x}-\{\gamma(1-x)-\delta x\} z=0
$$


and the result of differentiating this formula $n+1$ times is

$$
x(1-x) \frac{d^{n+2} z}{d x^{n+2}}+\{n+1-\gamma-(2 n+2-\gamma-\delta) x\} \frac{d^{n+1} z}{d x^{n+1}}-(n+1)(n-\gamma-\delta) \frac{d^{n} z}{d x^{n}}=0
$$

this shews that $d^{n} z / d x^{n}$ is a solution of the hypergeometric equation with elements $n+1$, $n-\gamma-\delta, n+1-\gamma$. This equation is a Riemann $P$-equation with singularities $0,1, \infty$ and exponents thereat $(0) 0, \gamma-n$; (1) $0, \delta-n ;(\infty) n+1, n-\gamma-\delta$.

Hence $y_{n}$ is a solution of the Riemann $P$-equation with singularities $0,1, \infty$ and exponents thereat $(0) \alpha, \alpha+\gamma-n$; (1) $\beta, \beta+\delta-n ;(\infty) n+1-\alpha-\beta, n-\alpha-\beta-\gamma-\delta$. An inspection of the general Riemann $P$-equation now enables us to see that

$$
x(x-1) \frac{d^{2} y_{n}}{d x^{2}}+\left\{a_{n}(x-1)+b_{n} x\right\} \frac{d y_{n}}{d x}+\left(E_{n}-\frac{\alpha A_{n}}{x}-\frac{\beta B_{n}}{1-x}\right) y_{n}=0
$$

where, for brevity, we have written

$$
\begin{array}{rlrl}
1-2 \alpha-\gamma+n & \equiv a_{n}, & 1-2 \beta-\delta+n & \equiv b_{n}, \\
\alpha+\gamma-n & \equiv A_{n}, & \beta+\delta-n & \equiv B_{n}, \\
(n+1-\alpha-\beta)(n-\alpha-\beta-\gamma-\delta) & \equiv E_{n} .
\end{array}
$$

Since we are about to integrate the differential equation (3), we naturally regard the two integrals

$$
\int_{0}^{1} \frac{y_{n} d x}{x} \equiv U_{n}, \quad \int_{0}^{1} \frac{y_{n} d x}{\mathrm{l}-x} \equiv V_{n}
$$

as additional standard forms. We shall also need an integral in which the integrand is the product of $y_{n}$ and a linear function of $x$; by taking this function to be $1-2 x$, so that we write

$$
\int_{0}^{1}(1-2 x) y_{n} d x \equiv J_{n}
$$

we introduce a combination of symmetry and skew-symmetry which more than compensates for formulae of greater length that would have been obtained if we had taken $x$ or $1-x$.

3. The result of integrating (3) as it stands is evidently

$$
\int_{0}^{1} x(x-1) \frac{d^{2} y_{n}}{d x^{2}} d x+\int_{0}^{1}\left\{\left(a_{n}+b_{n}\right) x-a_{n}\right\} \frac{d y_{n}}{d x} d x+E_{n} I_{n}-\alpha A_{n} U_{n}-\beta B_{n} V_{n}=0
$$

We eradicate the differential coefficients of $y_{n}$ from this result by performing partial integrations, two of the first term and one of the second. Hence we have

and this reduces to

$$
2 I_{n}-\left(a_{n}+b_{n}\right) I_{n}+E_{n} I_{n}-\alpha A_{n} U_{n}-\beta B_{n} V_{n}=0
$$

$$
\alpha A_{n} U_{n}+\beta B_{n} V_{n}=\left(E_{n}-a_{n}-b_{n}+2\right) I_{n}
$$

If we had multiplied (3) by $x$ before integrating, a similar piece of reduction would have yielded the result

$$
2 \beta B_{n} V_{n}=\left(E_{n}-2 \alpha A_{n}+2 \beta B_{n}-2 b_{n}+2\right) I_{n}-\left(E_{n}-2 a_{n}-2 b_{n}+6\right) J_{n} ;
$$

while the effect of multiplying (3) by $1-x$ would have been

$$
2 \alpha A_{n} U_{n}=\left(E_{n}+2 \alpha A_{n}-.2 \beta B_{n}-2 a_{n}+2\right) I_{n}+\left(E_{n}-2 a_{n}-2 b_{n}+6\right) J_{n} \text {. }
$$

Of course (9) is an immediate consequence of (10) and (11). 
We next consider $I_{n+1}$. Evidently

$$
y_{n+1}=x^{\alpha}(1-x)^{\beta} \frac{d\left\{y_{n} x^{-\alpha}(1-x)^{-\beta}\right\}}{d x}=\frac{d y_{n}}{d x}-\left(\frac{\alpha}{x}-\frac{\beta}{1-x}\right) y_{n}
$$

and consequently

so that

$$
I_{n+1}=\int_{0}^{1} \frac{d y_{n}}{d x} d x-\int_{0}^{1}\left(\frac{\alpha}{x}-\frac{\beta}{1-x}\right) y_{n} d x
$$

In like manner we find that

$$
I_{n+1}=\beta V_{n}-\alpha U_{n}
$$

$$
J_{n+1}=2(\alpha+\beta+1) I_{n}-\alpha U_{n}-\beta V_{n} .
$$

4. The required reduction formula is now obtained by combining the results of the preceding section. We first obtain a relation connecting $I_{n+1}, I_{n}, J_{n}$ by eliminating $U_{n}$ and $V_{n}$ from (10), (11) and (12); we transform this into a relation connecting $I_{n+2}, I_{n+1}, J_{n+1}$ by writing $n+1$ in place of $n$. Next we express $J_{n+1}$ in terms of $I_{n+1}$ and $I_{n}$ by eliminating $U_{n}$ and $V_{n}$ from (9), (12) and (13); we substitute this value of $J_{n+1}$ in the previous (transformed) relation, and the rest is a matter of simplification. We can substantially shorten some of the calculations by writing

$$
\alpha+\beta+\gamma+\delta \equiv 2 \sigma
$$

and (at a subsequent stage of the work)

$$
\left.\begin{array}{c}
\alpha+\beta-\gamma-\delta \equiv 2 \theta_{1}, \\
\alpha-\beta+\gamma-\delta \equiv 2 \theta_{2}, \\
\alpha-\beta-\gamma+\delta \equiv 2 \theta_{3} .
\end{array}\right\}
$$

The relation of the set $(\alpha, \beta, \gamma, \delta)$ to the set $\left(\sigma, \theta_{1}, \theta_{2}, \theta_{3}\right)$ is of a type which occurs in work on elliptic functions. It is a reciprocal relation, so that there is no difficulty in expressing a function of either set of numbers as a function of the other set. In particular we shall subsequently need the transformations

$$
\begin{aligned}
& E_{n}-a_{n}-b_{n}+2=\left(\sigma+\theta_{1}-n\right)(2 \sigma-n+1), \\
& E_{n}-2 a_{n}-2 b_{n}+6=\left(\sigma+\theta_{1}-n+1\right)(2 \sigma-n+2) \\
& (\alpha+\beta+1)\left(A_{n}+B_{n}\right)-\left(E_{n}-a_{n}-b_{n}+2\right)=(n+1)\left(\sigma-\theta_{1}-n\right) \text {, } \\
& 2 \alpha A_{n}-2 \beta B_{n}-a_{n}+b_{n}=\theta_{2}(4 \sigma-2 n+3)+\theta_{3}(2 \sigma-2 n+1)+2 \theta_{1} \theta_{2} . \\
& A_{n} B_{n} I_{n+1}+\left(A_{n}+B_{n}\right)\left(\alpha A_{n}-\beta B_{n}-\frac{1}{2} a_{n}+\frac{1}{2} b_{n}\right) I_{n} \\
& -\frac{1}{2}\left(A_{n}-B_{n}\right)\left(E_{n}-a_{n}-b_{n}+2\right) I_{n}+\frac{1}{2}\left(A_{n}+B_{n}\right)\left(E_{n}-2 a_{n}-2 b_{n}+6\right) J_{n}=0,
\end{aligned}
$$

and, when we replace $n$ by $n+1$, this can be written in the modified form

$$
\begin{aligned}
& (\alpha+\gamma-n-1)(\beta+\delta-n-1) I_{n+2}+\left(A_{n+1}+B_{n+1}\right)\left(\alpha A_{n+1}-\beta B_{n+1}-\frac{1}{2} a_{n+1}+\frac{1}{2} b_{n+1}\right) I_{n+1} \\
& \quad-\frac{1}{2}\left(A_{n+1}-B_{n+1}\right)\left(E_{n+1}-a_{n+1}-b_{n+1}+2\right) I_{n+1}+(\sigma-n-1)(2 \sigma-n+1)(\alpha+\beta-n) J_{n+1}=0 .
\end{aligned}
$$


Next we solve equations (12) and (13) for $\alpha U_{n}$ and $\beta V_{n}$, and insert the values so obtained in (9). The result is

$$
\frac{1}{2}\left(A_{n}+B_{n}\right) J_{n+1}=(\alpha+\beta+1)\left(A_{n}+B_{n}\right) I_{n}-\left(E_{n}-a_{n}-b_{n}+2\right) I_{n}-\frac{1}{2}\left(A_{n}-B_{n}\right) I_{n+1} ;
$$

this can be written in the modified form

$$
(\sigma-n) J_{n+1}=(n+1)(\gamma+\delta-n) I_{n}-\frac{1}{2}\left(A_{n}-B_{n}\right) I_{n+1} .
$$

When we multiply (20) by $\sigma-n$ and then replace $J_{n+1}$ by the expression supplied by (21), we obtain the required reduction formula in the form

$$
P_{n} I_{n+2}-Q_{n} I_{n+1}+R_{n} I_{n}=0
$$

with

$$
\begin{aligned}
& P_{n} \equiv(\sigma-n)(\alpha+\gamma-n-1)(\beta+\delta-n-1), \ldots \ldots \ldots \ldots \ldots \ldots \\
& R_{n} \equiv(n+1)(\sigma-n-1)(2 \sigma-n+1)(\alpha+\beta-n)(\gamma+\delta-n) \ldots . .
\end{aligned}
$$

Before we calculate the value of $Q_{n}$ in its simplest form, we make some observations on the kind of expression that we may expect to obtain for it. In the special cases (i) and (ii), described in $\S 1$, it is clear that, by reason of $(22), Q_{n} I_{n+1}$ vanishes whenever $n$ is an odd positive integer. Since $Q_{n}$ is a polynomial in $n$, we may consequently anticipate that it will vanish for all values of $n$ in these special cases. Now both $\theta_{3}$ and $\theta_{1}$ vanish in case (i), while both $\theta_{3}$ and $\theta_{2}$ vanish in case (ii). We are thus led to aim at expressing $Q_{n}$ as a sum of terms, each of which contains either $\theta_{3}$ or else the product $\theta_{1} \theta_{2}$ as a factor. It was with this aim in mind that we introduced $\theta_{1}, \theta_{2}, \theta_{3}$ in (15), and we now set about expressing $Q_{n}$ in terms of $\sigma, \theta_{1}, \theta_{2}, \theta_{3}$ instead of in terms of $\alpha, \beta, \gamma, \delta$.

The procedure by which (22) was derived from (20) and (21) makes it clear that $Q_{n}$ is equal to

$$
\begin{aligned}
\frac{1}{2}\left(A_{n+1}-\right. & \left.B_{n+1}\right)(\sigma-n)\left(E_{n+1}-a_{n+1}-b_{n+1}+2\right) \\
& -(\sigma-n)\left(A_{n+1}+B_{n+1}\right)\left(\alpha A_{n+1}-\beta B_{n+1}-\frac{1}{2} a_{n+1}+\frac{1}{2} b_{n+1}\right) \\
& +\frac{1}{2}\left(A_{n}-B_{n}\right)(\sigma-n-1)(2 \sigma-n+1)(\alpha+\beta-n) \\
=\theta_{2}(\sigma-n)( & 2 \sigma-n)\left(\sigma+\theta_{1}-n-1\right) \\
& -(\sigma-n)(\sigma-n-1)\left\{\theta_{2}(4 \sigma-2 n+1)+\theta_{3}(2 \sigma-2 n-1)+2 \theta_{1} \theta_{2}\right\} \\
& +\theta_{2}(\sigma-n-1)(2 \sigma-n+1)\left(\sigma+\theta_{1}-n\right) .
\end{aligned}
$$

Consider this expression $q u a$ function of $\theta_{1}, \theta_{2}, \theta_{3}$. It is a homogeneous linear function of $\theta_{2}, \theta_{3}, \theta_{1} \theta_{2}$ with coefficients which are functions of $n$ and $\sigma$. The coefficient of $\theta_{2}$ is

$$
(\sigma-n)(\sigma-n-1)\{(2 \sigma-n)-(4 \sigma-2 n+1)+(2 \sigma-n+1)\}=0 ;
$$

the coefticient of $\theta_{3}$ is

$$
-(\sigma-n)(\sigma-n-1)(2 \sigma-2 n-1) ;
$$

and, finally, the coefficient of $\theta_{1} \theta_{2}$ is

$$
(\sigma-n)(2 \sigma-n)-2(\sigma-n)(\sigma-n-1)+(\sigma-n-1)(2 \sigma-n+1)=(\sigma+1)(2 \sigma-2 n-1) .
$$

When we collect these results, we see that

$$
Q_{n}=(2 \sigma-2 n-1)\left\{\theta_{1} \theta_{2}(\sigma+1)-\theta_{3}(\sigma-n)(\sigma-n-1)\right\} .
$$


It is a simple matter to throw this formula into the alternative equivalent forms

$$
\begin{aligned}
& Q_{n}=(2 \sigma-2 n-1)\left\{\left(\theta_{1} \theta_{2}-\sigma \theta_{3}\right)(\sigma+1)+\theta_{3}(n+1)(2 \sigma-n)\right\}, \\
& Q_{n}=(2 \sigma-2 n-1)\left\{(\beta \gamma-\alpha \delta)(\sigma+1)+\theta_{3}(n+1)(2 \sigma-n)\right\} \ldots .
\end{aligned}
$$

We have now proved that $I_{n}$ satisfies the reduction formula (22) with $P_{n} ; Q_{n}$ and $R_{n}$ defined by (23), (27) and (24).

6. As was mentioned in $\S 1, I_{n}$ is easily expressible in terms of generalised hypergeometric functions, so that (22) yields a relation connecting three contiguous functions of this type. Such a relation proves to be less elegant than the relation connecting the numerators of the functions when they are expressed as quotients of polynomials in $\alpha, \beta, \gamma, \delta$. We calculate these numerators directly from (1).

We use the continental notation for binomial coefficients together with the notation for products which is now standard, namely

$$
(x)_{0} \equiv 1, \quad(x)_{r} \equiv x(x+1) \ldots(x+r-1), \quad(r \geqslant 1) .
$$

From the theorem of Leibniz it follows that

$$
\begin{aligned}
& I_{n}=\int_{0}^{1} x^{\alpha}(1-x)^{\beta}\left\{\sum_{r=0}^{n}\left(\begin{array}{l}
n \\
r
\end{array}\right) \frac{d^{n-r} x^{\gamma}}{d x^{n-r}} \cdot \frac{d^{r}(1-x)^{\delta}}{d x^{r}}\right\} d x \\
& =\sum_{r=0}^{n}(-)^{n-r}\left(\begin{array}{l}
n \\
r
\end{array}\right)(-\gamma)_{n-r}(-\delta)_{r} \int_{0}^{1} x^{\alpha+\gamma-n+r}(1-x)^{\beta+\delta-r} d x \\
& =\frac{\Gamma(\alpha+\gamma-n+1) \Gamma(\beta+\delta-n+1)}{\Gamma(2 \sigma-n+2)} H_{n} \text {, }
\end{aligned}
$$

where

$$
H_{n}=\sum_{r=0}^{n}(-)^{n-r}\left(\begin{array}{l}
n \\
r
\end{array}\right)(-\gamma)_{n-r}(\beta+\delta-n+1)_{n-r}(-\delta)_{r}(\alpha+\gamma-n+1)_{r} .
$$

In (22) we now replace $I_{n}$ by its value in terms of $H_{n}$, and we immediately get

$$
(2 \sigma-n)(\sigma-n) H_{n+2}-Q_{n} H_{n+1}+S_{n} H_{n}=0,
$$

where

$$
S_{n} \equiv(n+1)(\sigma-n-1)(\alpha+\beta-n)(\gamma+\delta-n)(\alpha+\gamma-n)(\beta+\delta-n) .
$$

Formula (29) has now been proved on the hypothesis that the real parts of $\alpha, \beta, \gamma, \delta$ are sufficiently large; but, since the formula asserts that a polynomial in $\alpha, \beta, \gamma, \delta$ is zero for such values of $\alpha, \beta, \gamma, \delta$, the polynomial must vanish identically. We have consequently proved that (29) holds for all values of $\alpha, \beta, \gamma, \delta$ without restriction when $H_{n}$ is defined by (28), while $Q_{n}$ and $S_{n}$ are defined by (27) and (30).

\section{WARWICK NEW ROAD \\ Leamington, WARWICKSHIRE}

